\title{
Extracorporeal Shockwave Therapy for the Treatment of Tendinopathies: Current Evidence on Effectiveness, Mechanisms, Limitations, and Future Directions
}

\author{
Ian Burton
}

Physiotherapist, Angus HSCP, NHS Tayside, Dundee, United Kingdom

Please cite as: Burton, I. (2020). Extracorporeal shockwave therapy for the treatment of tendinopathies: Current Evidence on Effectiveness, Mechanisms, Limitations, and Future Directions. SportRxiv doi: 10.31236/osf.io/fghaz

\begin{abstract}
Tendinopathy is a chronic degenerative tendon disorder which is characterised by pain, swelling and impaired physical function and performance, presenting in both athletes and the general population. Extracorporeal shockwave therapy (ESWT) is an increasingly common treatment for tendinopathy, which can initiate tendon healing and regeneration. The review presents current understanding of mechanisms of action of ESWT and provides a brief overview of its history and development. The central purpose of the review is to synthesise research findings investigating the effectiveness of ESWT for seven common tendinopathies (plantar
\end{abstract}


heel pain, rotator cuff, lateral elbow, Achilles, gluteal, hamstring and patellar tendinopathy) and provide recommendations on clinical applicability. Collectively, the available evidence indicates that ESWT is effective and can be recommended in treatment for the seven tendinopathies. However, the evidence is stronger for certain tendinopathies compared to others and uncertainties remain regarding the optimal ESWT treatment parameters. The consensus from recent literature is that although ESWT can be effective in isolation it should be combined with other treatments in tendinopathy, which needs to be addressed in future research.

Keywords: High-Energy Shock Waves; Tendinopathy; Resistance Training; Extracorporeal Shockwave Therapy; Musculoskeletal Diseases

\section{Introduction}

The recent global burden of disease study highlighted that musculoskeletal disorders are a leading cause of global disability and morbidity [1]. Tendinopathy is one of the most prevalent musculoskeletal disorders, responsible for $30-50 \%$ of all sports injuries [2]. Tendinopathy is a degenerative tendon disorder which is characterised by chronic pain, diffuse or localised swelling and impaired physical function and performance [3]. Tendons are susceptible to injury as they undergo high forces during repetitive sports activities, receive little vascular blood supply, have low elasticity and decreased metabolism compared to other tissues [4]. Histopathological findings in tendinopathy include collagen disorganisation and fibre separation, hypercellularity, increased mucoid ground substance and neovascularisation, without signs of inflammation [5]. However, the noninflammatory aetiology of tendinopathy has been challenged as inflammation is considered to play a key role in the initial phases of the disorder [6]. Recent European studies on lower limb tendinopathies estimate incidence and prevalence ranging from 7.0-11.8 and 10.5-16.6 per 1000 people, respectively $[7,8]$. The aetiology and pathogenesis of tendinopathy is considered multifactorial, resulting from a range of extrinsic and intrinsic factors [9]. Identified risk factors include impaired biomechanics [10], rheumatological and metabolic disorders [11], excess adiposity [12] fluoroquinolones [13]; genetics [14], and central nervous system hypersensitivity [15]. 
A range of approaches are used in tendinopathy rehabilitation including antiinflammatory medications, corticosteroid injections, laser therapy, ultrasound, platelet-rich plasma injections, prolotherapy, glycerol trinitrate patches, manual therapy, and various types of exercise [16]. Extracorporeal shockwave therapy (ESWT) is an emerging treatment option with a growing body of research investigating its applicability and effectiveness as a tendinopathy treatment [17, 18]. Due to its non-invasive nature, dearth of side effects, short duration and patient acceptability, ESWT offers a therapeutic rehabilitation method, when other conservative treatments are ineffective [19]. The purpose of this review is to synthesize the current available research on effectiveness of ESWT for seven tendinopathies (plantar heel pain, rotator cuff, lateral elbow, patellar, gluteal, hamstring and Achilles). The review will begin with an overview of the history of ESWT and its physical mechanisms, progressing to mechanisms of action for ESWT. Following an overview of the evidence on effectiveness of ESWT for tendinopathies, the review will conclude with considerations of the limitations of treatment parameters, future research needs and clinical recommendations.

\section{Methodology}

To conduct this narrative review, a systematic literature search was conducted using Medline/PubMed database to identify studies up to May 2020 using a combination of the following search terms: extracorporeal shockwave therapy, shock wave, shockwave therapy, shockwave, ESWT, tendon, tendinopathy, musculoskeletal, plantar, Achilles, patellar, rotator cuff, elbow, epicondylitis, gluteal, and hamstring. Relevant articles were retrieved and used to identify additional sources by cross-referencing and manually checking reference lists.

\section{ESWT history and development}

The effects of shockwaves on humans were first discovered during the Second World War, when German scientists discovered that shockwaves from a distant 
explosion could damage lung tissue [20]. In 1971 the first non-direct kidney stone disintegration was reported, leading to the development of the first clinical shockwave machines and human research [21]. The use of shockwaves in medicine began in Germany in 1980 with extracorporeal shockwave lithotripsy as a treatment to disintegrate renal and ureteric calculi [22]. In urological research, the discovery of new bone formation on pelvic bones was incidentally observed adjacent to the path of delivered shockwaves [23]. Consequently, further research identified the potential for both destructive and regenerative effects of shockwaves in human tissues [24]. Experimental research elucidated a dose-dependent response, with high-energy shockwaves being destructive and low-energy shockwaves causing regeneration in tissues [25]. Investigations into fracture healing concluded that better outcomes are achieved in treating bone non-unions, with ESWT successfully used as a treatment for non-union fractures in 1988 [26]. From the early 1990s the beneficial effects of ESWT on musculoskeletal disorders began emerging, leading to the development of focused ESWT (F-ESWT) devices, designed specifically to treat musculoskeletal disorders [27]. The destructive potential of high-energy ESWT was first applied to rotator cuff tendinopathies of the shoulder with extraosseous calcification, allowing for calcific deposit disintegration [28]. Next, low-energy F-ESWT was tested with other chronic tendinopathies, beginning with plantar fasciopathy and then lateral elbow tendinopathy, showing beneficial regenerative effects [29]. In the early 2000s new devices with ballistic pressure waves were introduced, referred to as radial ESWT (R-ESWT), with clinical effectiveness found for superficial musculoskeletal disorders [30]. Today ESWT is a widely accepted clinical treatment, which is applied to a broad range of musculoskeletal disorders and is considered a significant innovation in regenerative medicine [31].

\section{Physical mechanisms of shockwaves}

Shockwaves can be defined as mechanical acoustic energy waves, with positive pressure followed by negative pressure, and returning to ambient values within microseconds [32]. Shockwaves are sonic pulses characterised by high peak pressure amplitudes of up to $100 \mathrm{mpa}$ (500 bar), with rapid pressure rise times 
under 10 nanoseconds, a short lifespan of under 10 microseconds and a broad frequency range, usually 16-20 $\mathrm{MHz}$ [33]. Because of the rapid pressure increases, shockwaves have been compared to 'micro explosions' [33]. High pressure shockwaves produced in the positive phase can be reflected or absorbed, whereas the negative phase causes formation of cavitation bubbles due to negative pressure at tissue interfaces [34]. Cavitation bubbles implode at high speed, generating subsequent shockwaves or fluid microjets, which stimulate direct and indirect effects on tissues [35]. The peak pressure generated by shockwaves are typically 1000 times greater than that created by therapeutic ultrasound [19]. Focused shockwaves are typically generated through a fluid medium, by either an electrohydraulic, piezoelectric, or electromagnetic type source generator [32]. Electro-pneumatically generated radial shockwaves do not technically form shockwaves, rather radial pressure waves, which can induce acoustic cavitation via a ballistic mechanism [36]. Compressed air accelerates a projectile, typically a bullet inside a cylindrical handpiece. When the projectile hits an applicator at the end of the tube, high-energy pressure waves are produced which radially expand from the skin into tissue in spherical waves [34]. Unlike F-ESWT, the waves are not focused into small deeper tissues, therefore are suitable for treating large superficial tissues [36]. Despite these differences, each generator type focuses pressure impulses into the targeted tissue which mechanically stimulate cells through mechano-transduction, activating biochemical signalling and tissue regeneration [37].

\section{General mechanisms of action}

Despite evidence indicating effectiveness of ESWT across a plethora of medical conditions, its mechanisms of action remain not fully understood. Haupt [25] proposed four possible phase mechanisms of ESWT effects on tissue: physical, physicochemical, chemical, and biological. In the physical phase, shockwave pressures cause absorption, reflection, refraction, and transmission of energy into cells. These tensile forces induce cavitation, increasing cell membrane permeability and activating cell signalling pathways [33]. These include mechanotransduction pathways and those that regulate a variety of gene expressions [38]. 
In the physicochemical phase cells release molecules such as adenosine triphosphate (ATP), activating cell signalling pathways [39]. In the chemical phase, ion channels in cell membranes are altered allowing cell calcium mobilization [40]. The biological phase plays host to a plethora of identified biological responses due to tissue and cell stimulation [41]. Tissue-healing responses are mediated by release of vascular endothelial growth factor (VEGF), endothelial progenitor cells, endothelial nitric oxide synthase (eNOS), insulin-like growth factor (IGF-1) proliferating cell nuclear antigen (PCNA) and various other functional proteins $[31,42]$. Cellular modulation is activated by upregulation and downregulation of proteins which promotes processes such as neovascularization [43], anti-inflammation [44], anti-apoptosis [45], chondroprotective effects [46], angiogenesis [47], and regeneration of bone and tendon tissue [48]. Additionally, ESWT activates immune system responses [49] and anti-inflammation by stimulating macrophage, T-cell, and mesenchymal stem cell proliferation [50-52). ESWT also modulates expression of inflammatory cytokines such as interleukin-6, interleukin-10 and tumour necrosis factor alpha, leading to anti-inflammatory effects [53].

Another proposed mechanism of ESWT on mechanobiology is its influence on the nervous system and neurophysiological processes. Research has reported analgesic effects of ESWT, which may be due to modulating neurological responses, although exact mechanisms are unknown [54]. However, ESWT has been shown to modulate the presence or function of unmyelinated nerve fibres and pain neurotransmitters such as substance $P$ [55] and calcitonin gene-related peptide [56]. Nociceptor hyperstimulation and stimulation of nociceptive C-fibres can also release pain inhibiting substances such as neuropeptides [57]. Studies have also shown the potential of ESWT in reducing hypertonia and spasticity and regenerative effects on nerves and spinal injuries [58, 59]. Wess [60] identified how ESWT could reorganise pathological memory pathways leading to permanent pain relief, due to the mitigation of pain central sensitization, which is believed to play a key role in chronic pain experienced in tendinopathy. Recently transcranial F-ESWT was found to increase vigilance in those with unresponsive wakefulness syndrome and improve symptoms in polyneuropathy [61]. Further research is 
required to determine the exact neurophysiological mechanisms exerted by ESWT and their full potential.

\subsection{Tendon specific mechanisms of action}

Chronic tendinopathies have historically had poor outcomes with traditional treatment methods, however ESWT may exert a plethora of direct healing effects on tendon tissue during treatment [62]. Treatment with ESWT can influence cell homeostasis and tissue regeneration by stimulating healing responses, such as inducing proliferation, differentiation, and migration of mesenchymal stem cells [63]. However, shockwaves can target many cell types, including stromal cells, endothelial cells, osteoblasts, fibroblasts and tendon specific tenocytes and collagen [31]. In vitro studies have also found shockwaves activate tendon repair processes by increasing normal fibroblast proliferation and activating gene expression for Transforming growth factor beta 1 and for collagen types I and III [64]. Several other tendon specific responses have been observed, including reduction in matrix metalloproteinases and inflammatory cytokines, and upregulation of tendon cells and anti-inflammatory cytokines [53]. There is also proliferation and migration of proteins involved in collagen synthesis and tenocytes in damaged tendons and increased lubricin expression, stimulating healing $[65,66]$. The mechanical stimulus of ESWT has been postulated to generate tendinopathy collagen synthesis and remodelling by promoting catabolic and inflammatory processes and stimulating removal of pathological matrix constitutes [48]. The following sections will summarise the evidence of clinical effectiveness of ESWT for specific tendinopathies.

\section{Clinical effectiveness of ESWT}

\subsection{Rotator cuff tendinopathy}

Rotator cuff tendinopathy is thought to be caused by a combination of extrinsic and intrinsic mechanisms leading to chronic tendon degeneration, although its 
aetiology is not fully understood [9]. The disorder is characterised by persistent pain, disability and weakness in the shoulder, especially when lifting overhead or abducting the arm beyond 90 degrees [67]. Prevalence is estimated to be as high as $14 \%$ in the general population [68] and has been reported as high as $30 \%$ in volleyball players [69]. Rotator cuff tendinopathy can lead to long-term disability, with up to $42 \%$ reporting symptoms after ten years [70]. Calcific rotator cuff tendinopathy is thought to be caused by repetitive mechanical overloading leading to calcium hydroxyapatite deposition, with $80 \%$ of cases involving the supraspinatus tendon [71]. Although eccentric exercise can be effective [72], ESWT has emerged as an alternative treatment option prior to surgery [73] and has been found equally effective as supervised exercise [74]. Several systematic reviews and meta-analysis have been published on ESWT and rotator cuff tendinopathy, with similar positive findings [75-85].

Huisstede et al. [75] concluded that only high-energy ESWT is effective for calcific rotator cuff tendinopathy, with no evidence for non-calcific rotator cuff tendinopathy. Similarly, Bannuru et al. [76] concluded high-energy ESWT is effective for improving function and pain in calcific rotator cuff tendinopathy and can result in complete calcification resolution, unlike low-energy ESWT or placebo. A meta-analysis by Verstraelen et al [77] also found high-energy ESWT better at improving function and calcific reabsorption compared to low-energy ESWT. A recent comprehensive systematic review and network meta-analysis comparing all non-surgical treatment for calcific rotator cuff tendinopathy included 14 RCTs and concluded that F-ESWT is the best treatment for improving pain, function and calcium deposit resolution [78]. Several other reviews also concluded that ESWT is safe and effective in calcific rotator cuff tendinopathy, with ESWT improving shoulder function, reducing pain and calcific deposits, with efficacy maintained at 6-month follow-up [79-81]. Several high quality randomized controlled trials (RCTs) as assessed by these reviews have found significant benefit of ESWT in calcific rotator cuff tendinopathy [28, 82-84]. Despite compelling evidence and recommendations for calcific rotator cuff tendinopathy, none of these reviews or others recommend ESWT for non-calcific rotator cuff tendinopathy [85]. Several RCTs have not found any benefit, therefore its use can be controversial and often not recommended $[86,87]$. However, many of these studies fail to clarify the 
exact clinical diagnosis in patients and satisfactory results have been found in RCTs with strict inclusion criteria and clinical diagnosis for non-calcific rotator cuff tendinopathy [88].

\subsection{Lateral elbow tendinopathy}

Lateral elbow tendinopathy is caused by repetitive microtrauma of the extensor carpi radialis brevis muscle and tendon leading to chronic degeneration [89]. The disorder has been previously referred to as 'tennis elbow' or 'Lateral epicondylitis', suggesting an inflammatory disorder [90]. Symptoms include lateral elbow pain which can radiate down the arm and weak grip strength [91]. Recreational sports, increased age, smoking, diabetes, repetitive work tasks using the hands or wrists are risk factors, with prevalence as high as $2.8 \%$ in the general population [92] and up to $13.1 \%$ in climbing athletes [93]. Eccentric exercise has shown good outcomes for improving pain and function and is recommended as first-line conservative treatment [94]. However, exercise applied in isolation is considered to have low long-term effectiveness, suggesting other more effective treatments are warranted [95]. Although ESWT has been shown to be an efficacious treatment for lateral elbow tendinopathy, it is not without controversy as some RCTs have provided conflicting results [96-100].

A systematic review of nine RCTs by Buchbinder et al. [101] concluded there was high level evidence that ESWT provides little or no benefit for pain and function in lateral elbow tendinopathy. A systematic review by Rompe and Maffulli [102] including ten RCTs also acknowledged conflicting results, although the authors identified significant methodological heterogeneity and flaws throughout the studies. Only five RCTs had high quality placebo-controlled methodology, two of which reported significant success of ESWT over placebo [103, 104]. Three other high-quality trials not finding benefit still had important quality deviations in their study design from the previous two [105-107]. Three lower quality treatmentcontrolled trials reported effectiveness of ESWT [108-112]. Despite methodological heterogeneity, the authors concluded there was effectiveness of 
ESWT for lateral elbow tendinopathy when well-defined restrictive study parameters are appropriately implemented. A recent systematic review and metaanalysis included 13 studies and 1035 patients comparing ESWT with other treatments for lateral elbow tendinopathy [113]. Meta-analysis showed that ESWT was superior for pain and function (loss of grip strength) compared to other treatment methods. Another recent meta-analysis including 12 studies concluded that R-ESWT was superior to F-ESWT for pain and function (grip strength) but only at short-term follow-up of 24-weeks [114]. Similarly, a meta-analysis including 5 RCTs comparing ESWT with ultrasound, found superiority of ESWT for pain and grip strength improvement at one, three and six months [115]. Another meta-analysis including 4 studies comparing ESWT with corticosteroid injections, found ESWT superior for pain and grip strength at 12 weeks [116].

\subsection{Patellar tendinopathy}

Patellar tendinopathy or 'jumpers' knee' results from repetitive microtrauma to the patella tendon causing an initial inflammatory response progressing to chronic degeneration [4]. Prevalence in the general population is around 14\% [117], and as high as $30-45 \%$ in elite jumping athletes such as basketball and volleyball players, due to repetitive jumping and excessive knee loading [118]. Despite being challenging to treat, eccentric loading exercise such as decline squats can be useful for management of the disorder [119]. However, ESWT has been found more effective than stretching and strengthening exercises for the quadriceps and hamstrings [120]. Mani-Babu et al. [17] conducted a systematic review including seven studies on ESWT for patellar tendinopathy [120-126]. It concluded that ESWT is a promising short and long-term treatment for patellar tendinopathy, although the evidence is limited and further RCTs with robust design are needed. A systematic review by Korakakis et al. [18] concluded there was moderate-level evidence that ESWT is no more effective than placebo in short and mid-term for patellar tendinopathy. The authors also concluded there was low level evidence that ESWT is superior to standard conservative treatment in long-term outcomes. The review included 5 studies [120, 124, 127-129], with three [120, 124, 129] having significant methodological flaws, and the other two did not compare ESWT 
with placebo control $[127,128]$. Despite claiming to use robust methods and quality assessment tools, high quality ESWT studies were not included, which may explain the inconsistent results. The authors acknowledged the need for methodological standardisation, suitability of quality assessment tools and reporting guidelines in future ESWT study design [18].

Despite ESWT having generally positive outcomes for patellar tendinopathy, studies have used extremely variable treatment parameters, leading to controversies regarding treatment protocols, effectiveness and recommendations [130]. There have been conflicting findings in RCTs investigating ESWT for patellar tendinopathy, with some finding no significant differences [124, 127, 129]. However, the studies reporting poor results have been rife with methodological flaws, questioning their reliability [131]. All the RCTs with robust methodological design have found positive effects of ESWT, quantifying the recommendations made by Mani-Babu et al. [17] and others [117] recommending its use for patellar tendinopathy. Two recent systematic reviews and meta-analysis comparing all treatments for patellar tendinopathy, both concluded that platelet-rich-plasma injections were the most superior treatment but did acknowledge that ESWT was effective for pain and function to a lesser extent $[132,133]$.

\subsection{Achilles tendinopathy}

Achilles tendinopathy is caused by repetitive microtrauma of the Achilles tendon and failed healing, leading to chronic degeneration, with evidence of inflammation being histologically absent [134]. Intrinsic and extrinsic risk factors include impaired biomechanics, overtraining, high body mass index, fluoroquinolone use, and history of metabolic or inflammatory disorders [135, 136]. Symptoms including pain, disability and tendon swelling typically last six months with up to $15 \%$ being symptomatic five years after diagnosis [137]. Achilles tendinopathy is common in athletes, particularly runners, with prevalence reported as high as $12.5 \%$ in adult athletes [138] and $7.8 \%$ in adolescent runners [139]. However up to $30 \%$ of cases present in those who do not participate in sports activities [140]. 
Heavy slow resistance training and eccentric exercise such as the 'Alfredson protocol' have shown good outcomes [141]. However, progressive eccentric exercise has been found less effective than ESWT alone for chronic insertional Achilles tendinopathy [142]. In unresponsive cases surgery may be required, however ESWT has emerged as a potential alternative treatment prior to surgery [143].

The systematic review by Mani-Babu et al. [17] included 11 studies on Achilles tendinopathy, four with midportion Achilles tendinopathy [144-147], two with insertional Achilles tendinopathy [142, 148], and five with both [149-153]. Despite some conflicting findings from studies comparing ESWT to sham [149], the results indicated overall that ESWT is an effective short-term intervention for pain and function in Achilles tendinopathy and should be recommended in clinical practice. There is also some low-quality evidence for long-term effectiveness [17]. Another systematic review concluded there was moderate evidence for F-ESWT in midportion and insertional Achilles tendinopathy [85]. The systematic review by Korakakis et al. [18] concluded there was low level evidence that ESWT is comparable to eccentric exercise and superior to no treatment for midportion Achilles tendinopathy and superior to eccentric exercise for insertional Achilles tendinopathy. Other systematic reviews have concluded that while ESWT is an effective treatment for pain and function, it may not be superior to eccentric exercise and may be more effective when combined with it [154-156]. Despite overall good evidence, all these reviews acknowledged that longer-term followups from higher quality RCTs with strict study design are needed to build upon the evidence for Achilles tendinopathy.

\subsection{Plantar heel pain}

Plantar heel pain (PHP) is thought to be caused by repetitive microtrauma leading to degenerative shortening of the plantar fascia collagen matrix and possible heel spur formation [157]. The previously used diagnosis 'plantar fasciitis' suggested an inflammatory origin, and although inflammatory cells are present, chronic 
degeneration is considered the primary cause of the condition [158]. Moreover, the term 'plantar fasciopathy' is considered a more accurate diagnosis describing the chronic degenerative changes of the fascia, with many experts considering the condition to present like a tendinopathy [157]. Symptoms include heel pain in the morning and after activity or rest, and functional limitations such as impaired gait and reduced physical activity [159]. PHP has a lifetime prevalence of $10 \%$ in the general population [160], with prevalence ranging from $5.2 \%$ to $17.5 \%$ in running athletes [161]. A variety of treatment approaches have shown good short-term outcomes for PHP, including plantar-fascia specific stretching and resistance training either in isolation or combined with corticosteroid injections [162]. However, due to inadequate long-term outcomes of treatment, more effective treatments are required $[163,164]$. Several systematic reviews and metaanalyses have concluded there is long-term safety and efficacy of ESWT, recommending its use for PHP [165-180].

A systematic review by Rompe et al. [165] included all 17 RCTs on F-ESWT for PHP published at that time, five of which were determined to be of good quality. They highlighted significant heterogeneity in study design, perhaps due to their limited inclusion criteria stating meta-analysis was inappropriate. Despite concluding there was a preponderance of well-designed studies, it recommended ESWT after traditional conservative methods had failed. Following this review, higher quality RCTs with robust methodological design began emerging [166]. A systematic review and meta-analysis by Chang et al. [167] of 12 studies including 11,431 patients compared the effectiveness of F-ESWT of different intensities and R-ESWT for PHP. It concluded that using the highest most tolerable energy output within medium intensity ranges is the most superior F-ESWT method and that RESWT is a cost-effective efficacious alternative. A systematic review including ten studies on PHP concluded that overall, there is good Level-1 evidence for the effectiveness of F-ESWT, with one study providing Level-1 evidence for R-ESWT [85]. A meta-analysis by Aqil et al. [168] included seven RCTs comparing ESWT with placebo. It concluded that ESWT is safe and effective for PHP unresponsive to other nonoperative treatments, with improved pain scores after 12-weeks and maintained for 12 months. It recommended the use of ESWT for PHP pain, with a minimum of three months failed conservative treatment. A meta-analysis by 
Zhiyun Li et al. [169] on F-ESWT and PHP with strict inclusion criteria, included five RCTs. It concluded there was strong evidence that ESWT was effective in treating PHP compared to placebo and recommended its use when conservative interventions fail. A meta-analysis by Dizon et al. [170] included 11 high quality RCTs and concluded that both moderate and high-energy ESWT are effective in treating PHP. A systematic review and meta-analysis by Yin et al. [171] included seven RCTs and concluded ESWT was significantly superior for pain relief and function over control for PHP. It recommended that studies with long-term followup are needed to elucidate long-term efficacy. A meta-analysis by Lou et al. [172] included nine RCTs comparing ESWT without local anaesthesia against placebo in PHP treatment. ESWT significantly improved success rates of reduced heel pain, reducing pain by $60 \%$ with morning pain and during activities. It concluded ESWT is particularly effective in relieving PHP pain and recommended its use when traditional treatments have failed.

A methodologically robust meta-analysis by Sun et al. [173] included nine high quality RCTs on ESWT and PHP. It concluded that F-ESWT is associated with higher success rates and pain reduction compared to sham in PHP, recommending it when conservative treatment fails. However, the authors acknowledged limitations of the included studies, suggesting further high-quality RCTs with larger sample sizes are warranted. Recently, several systematic reviews with network meta-analysis have compared ESWT versus other conservative PHP treatments, with all finding superior outcomes for ESWT. A recent comprehensive systematic review with network meta-analysis included 31 RCTs comparing effectiveness of all commonly used PHP treatments, with ESWT being ranked the most effective treatment for short, medium and long-term pain and function [174]. A network meta-analysis including 19 RCTs and 1676 participants compared ESWT ultrasound, ultrasoundguided pulsed radiofrequency, intracorporeal pneumatic shock therapy, LLLT and non-invasive interactive neurostimulation for PHP pain relief [175]. Only R-ESWT induced significant pain reduction compared with placebo at six weeks and was the only recommended treatment. Another recent network meta-analysis compared pain relief performance of eight different therapies, including 41 studies and 2,880 patients. ESWT was superior to nonsteroidal anti-inflammatory medications, corticosteroid injections, autologous whole blood, platelet-rich 
plasma, ultrasound therapy, botulinum toxin A, and dry needling. In terms of onemonth, three-month and six-month pain outcomes, only ESWT turned out to be of better efficacy than placebo. For all seven outcomes ESWT ranked first and was recommended as the optimal treatment [176]. Systematic reviews and metaanalysis have also directly compared ESWT versus corticosteroid injections [177179], and ultrasound [180], with all finding ESWT superior.

\subsection{Gluteal tendinopathy}

The diagnosis of 'greater trochanteric pain syndrome' has been associated with trochanteric bursitis and inflammation of gluteal tendons [181]. However, the aetiology of gluteal tendinopathy is associated with the degeneration of the gluteal tendons attaching to the greater trochanter [182]. The combination of excessive compression and high tensile loads within these tendons may cause damage [183]. Lateral hip pain with resisted hip abduction, passive adduction and palpation can confirm diagnosis, with hip muscle weakness, altered biomechanics and impaired gait also common [184]. Prevalence in those over age 50 has been reported as high as $18 \%$ and is highest in middle aged women, impacting on daily activities, quality of life and causing psychological distress [185]. Exercise and load modification can be successful, but recurrence is common and refractory cases can require surgery [186]. A systematic review of 14 studies on gluteal tendinopathy management concluded there was moderate evidence for ESWT, however they identified a need for better quality RCTs [187]. Similarly, another systematic review on ESWT and lower limb tendinopathy concluded there was moderate evidence ESWT was more effective than other non-operative interventions, recommending it as an alternative treatment [17]. These reviews included the only two good quality RCTs conducted on gluteal tendinopathy, with ESWT composite effectiveness determined to be $78.8 \%$ at 12 months [188] and $74 \%$ at 15 months [189]. The most recent RCT conducted on gluteal tendinopathy compared F-ESWT with ultrasound in 50 patients, including 44 females [190]. Treatment with F-ESWT was significantly superior to ultrasound for reducing pain measured by p-NRS at both two months (2.08 vs 3.36) and six months (0.79 vs 2.03). There was a significant improvement in physical function in both groups, 
with neither being statistically superior. Despite these positive findings, the current evidence is limited with further high-quality RCTs needed.

\subsection{Hamstring tendinopathy}

Proximal hamstring tendinopathy can occur in both the general population and in athletes involved in sports with frequent change of direction movements such as soccer or sagittal plane activities such as hurdling or sprinting [191]. It is not uncommon for the condition to present bilaterally and it is characterised by localized deep pain in the ischial tuberosity region that is often exacerbated after activities such as running, squatting or sitting [192]. There is currently a lack of consensus regarding diagnostic criteria and treatment, with progressive exercise considered the standard treatment approach [193]. Any of the hamstring tendons can be involved, with the biceps femoris tendon (41\%) the most implicated [194]. Compression of the tendon at its attachment to the ischial tuberosity during hip flexion and adduction is considered a key etiological factor, leading the condition to be considered as an insertional tendinopathy [195]. The only RCT investigating ESWT for hamstring tendinopathy was conducted with 40 professional athletes, with ESWT compared against conventional physiotherapy including an exercise program over one month [196]. At 3 months after treatment, 17 of the 20 patients $(85 \%)$ in the ESWT group and two of the 20 patients (10\%) in the physiotherapy group achieved a reduction of at least $50 \%$ in pain. The authors concluded that ESWT was safe and effective for pain and function in hamstring tendinopathy and was superior to conventional physiotherapy at three, six and twelve months.

\section{Controversy and Limitations}

Despite the theoretical plausibility and clinical success of ESWT in tendinopathy, a myriad of treatment protocols have been used in studies, with vast variances in treatment delivery methods [197]. Some studies have found no benefit of ESWT; therefore, some controversy exists surrounding its use. However, these poor 
results may be related to differences in methodology, patient section criteria, device used, energy levels and outcome measures [19]. There are wide variances in treatment sessions used, with some recommending one session and others a standardized model of three sessions [198]. An individualized protocol adapted to patient response, with as many as 11 sessions has also been found feasible and recommended over standardised protocols [199]. Studies have also shown that ESWT is less effective for tendinopathy when administered with local anaesthesia as it inhibits analgesic effects and release of pain mediators with hyperstimulation [172]. The conflicting findings in studies using ESWT for tendinopathy may be explained by variances in study design (patient number, chronic or acute condition, sham group, comparisons with other treatment). Treatment parameters also differ (total energy use, R-ESWT or F-ESWT, low-energy, high-energy, direct or indirect contact, device differences). Differences in outcomes measures are also common (follow-up duration, pain assessment method, use of an algometer or a dolorimeter, objective or subjective assessment of pain, success rate measurement). Controversy over the ESWT type, energy, number of treatment sessions, use of image-guided or clinically guided methods, and the area undergoing ESWT has resulted in uncertainty regarding optimal treatment parameters [166]. These issues should be addressed in future research in order to elucidate the optimal treatment protocols.

In clinical practice, ESWT is rarely used in isolation and is often combined with specific exercises, however, RCTs have generally not adequately addressed this, often comparing ESWT alone with placebo [200]. As monotherapy is not often implemented clinically, there is a need for a comparison of more applicable treatment interventions in future ESWT studies [201]. Current research recommends combined rather than isolated treatments in tendinopathy, and in clinical practice ESWT is often combined with exercise during treatment [202]. However, the majority of RCTs investigating ESWT have used it in isolation, which is not reflective of clinical practice. Recently some studies have found combining ESWT and specific exercise feasible and effective for tendinopathy [147]. However, the optimal treatment protocols for ESWT interventions in isolation or combined with exercise are still undetermined [197]. There is increasing evidence for the clinical effectiveness of ESWT in tendinopathy, but there is still a need for 
larger and higher quality studies [85]. The most effective treatment protocols need to be determined with future research following clinical practice, where treatment is delivered as comprehensive protocols.

\section{Conclusion and recommendations}

This review has provided a comprehensive overview of the research to date on ESWT for tendinopathy. Although there are uncertainties regarding optimal treatment parameters and protocols for ESWT in tendinopathy treatment, there is clear evidence of effectiveness for improving pain and function in the seven tendinopathies reviewed. Therefore, ESWT can be recommended as a treatment for PHP, Achilles, patellar, lateral elbow and calcific rotator cuff tendinopathies based on a significant body of evidence including systematic reviews and metaanalysis. Despite the dearth of studies for hamstring and gluteal tendinopathy, RCTs have shown good outcomes and while ESWT can be recommended, further substantive high-quality studies are required in order to make definitive recommendations. When implemented for tendinopathy, ESWT should be administered following currently recommended protocols and parameters to increase likelihood of effectiveness. Further research is required to determine the optimal ESWT protocols for specific tendinopathies, with future studies investigating comprehensive treatment interventions which combine ESWT with other tendinopathy treatments such as exercise, reflective of clinical practice.

Funding: No sources of funding were used to assist in the preparation of this article.

Conflicts of interest/Competing interests: The author declares no conflicts of interest relevant to the content of this review.

Authorship contributions: IB conceptualised the work, wrote the first draft of the manuscript, revised the manuscript and approved the final manuscript. 


\section{References}

1. Sebbag E, Felten R, Sagez F, Sibilia J, Devilliers H, Arnaud L. The world-wide burden of musculoskeletal diseases: a systematic analysis of the World Health Organization Burden of Diseases Database. Ann Rheum Dis 2019;78:844-8.

2. Mead MP, Gumucio JP, Awan TM, Mendias CL, Sugg KB. Pathogenesis and Management of Tendinopathies in Sports Medicine. Transl Sports Med 2018;1:513.

3. Ackermann PW, Renstrom P. Tendinopathy in sport. Sports Health 2012;4:193201.

4. Malliaras $P$, Barton CJ, Reeves ND, Langberg $H$. Achilles and patellar tendinopathy loading programmes : a systematic review comparing clinical outcomes and identifying potential mechanisms for effectiveness. Sports Med $2013 ; 43: 267-86$.

5. Magnusson SP, Langberg $H$, Kjaer $M$. The pathogenesis of tendinopathy: balancing the response to loading. Nat Rev Rheumatol 2010;6:262-8.

6. Millar $\mathrm{NL}$, Hueber $\mathrm{AJ}$, Reilly $\mathrm{JH}$, et al. Inflammation is present in early human tendinopathy. Am J Sports Med 2010;38:2085-91.

7. Riel $\mathrm{H}$, Lindstrom CF, Rathleff MS, Jensen MB, Olesen JL. Prevalence and incidence rate of lower-extremity tendinopathies in a Danish general practice: a registry-based study. BMC Musculoskelet Disord 2019;20:239,019-2629-6.

8. Albers IS, Zwerver J, Diercks RL, Dekker JH, Van den Akker-Scheek I. Incidence and prevalence of lower extremity tendinopathy in a Dutch general practice population: a cross sectional study. BMC Musculoskelet Disord 2016;17:16,0160885-2.

9. Seitz AL, McClure PW, Finucane S, Boardman ND,3rd, Michener LA. Mechanisms of rotator cuff tendinopathy: intrinsic, extrinsic, or both? Clin Biomech (Bristol, Avon) $2011 ; 26: 1-12$. 
10. Sprague AL, Smith AH, Knox P, Pohlig RT, Gravare Silbernagel K. Modifiable risk factors for patellar tendinopathy in athletes: a systematic review and metaanalysis. Br J Sports Med 2018;52:1575-85.

11. Abate M, Schiavone C, Salini V, Andia I. Occurrence of tendon pathologies in metabolic disorders. Rheumatology (Oxford) 2013;52:599-608.

12. Scott A, Zwerver J, Grewal N, et al. Lipids, adiposity and tendinopathy: is there a mechanistic link? Critical review. Br J Sports Med 2015;49:984-8.

13. Alves C, Mendes D, Marques FB. Fluoroquinolones and the risk of tendon injury: a systematic review and meta-analysis. Eur J Clin Pharmacol $2019 ; 75: 1431-43$.

14. Collins M, Raleigh SM. Genetic risk factors for musculoskeletal soft tissue injuries. Med Sport Sci 2009;54:136-49.

15. Plinsinga $M L$, Brink MS, Vicenzino $B$, van Wilgen $C P$. Evidence of Nervous System Sensitization in Commonly Presenting and Persistent Painful Tendinopathies: A Systematic Review. J Orthop Sports Phys Ther 2015;45:86475.

16. Cardoso TB, Pizzari T, Kinsella R, Hope D, Cook JL. Current trends in tendinopathy management. Best Pract Res Clin Rheumatol 2019;33:122-40.

17. Mani-Babu S, Morrissey D, Waugh C, Screen H, Barton C. The effectiveness of extracorporeal shock wave therapy in lower limb tendinopathy: a systematic review. Am J Sports Med 2015;43:752-61.

18. Korakakis V, Whiteley R, Tzavara A, Malliaropoulos N. The effectiveness of extracorporeal shockwave therapy in common lower limb conditions: a systematic review including quantification of patient-rated pain reduction. $\mathrm{Br}$ J Sports Med 2018;52:387-407.

19. Wang CJ. Extracorporeal shockwave therapy in musculoskeletal disorders. J Orthop Surg Res 2012;7:11,799X-7-11. 
20. Chaussy C, Eisenberger F, Forssmann B. Extracorporeal shockwave lithotripsy (ESWL): a chronology. J Endourol 2007;21:1249-53.

21. Chaussy C, Brendel W, Schmiedt E. Extracorporeally induced destruction of kidney stones by shock waves. Lancet $1980 ; 2: 1265-8$.

22. Chaussy C, Wilbert DM. Extracorporeal shockwave lithotripsy today--an assessment of current status. Urologe A 1997;36:194-9.

23. Graff J, Diederichs W, Schulze H. Long-term followup in 1,003 extracorporeal shock wave lithotripsy patients. J Urol 1988;140:479-83.

24. Ikeda K, Tomita K, Takayama K. Application of extracorporeal shock wave on bone: preliminary report. J Trauma 1999;47:946-50.

25. Haupt G. Use of extracorporeal shock waves in the treatment of pseudarthrosis, tendinopathy and other orthopedic diseases. J Urol 1997;158:411.

26. Wang CJ, Chen HS, Chen CE, Yang KD. Treatment of nonunions of long bone fractures with shock waves. Clin Orthop Relat Res 2001;(387):95-101.

27. Krischek O, Rompe JD, Herbsthofer B, Nafe B. Symptomatic low-energy shockwave therapy in heel pain and radiologically detected plantar heel spur. $Z$ Orthop Ihre Grenzgeb 1998;136:169-74.

28. Gerdesmeyer L, Wagenpfeil S, Haake M, et al. Extracorporeal shock wave therapy for the treatment of chronic calcifying tendonitis of the rotator cuff: a randomized controlled trial. JAMA 2003;290:2573-80.

29. Rompe JD, Eysel P, Hopf C, et al. Extracorporeal shockwave therapy in orthopedics. Positive results in tennis elbow and tendinosis calcarea of the shoulder. Fortschr Med 1997;115:26, 29-33.

30. Foldager CB, Kearney C, Spector M. Clinical application of extracorporeal shock wave therapy in orthopedics: focused versus unfocused shock waves. Ultrasound Med Biol 2012;38:1673-80. 
31. d'Agostino MC, Craig K, Tibalt E, Respizzi S. Shock wave as biological therapeutic tool: From mechanical stimulation to recovery and healing, through mechanotransduction. Int J Surg 2015;24:147-53.

32. Gerdesmeyer L, Maier M, Haake M, Schmitz C. Physical-technical principles of extracorporeal shockwave therapy (ESWT). Orthopade 2002;31:610-7.

33. Ogden JA, Toth-Kischkat A, Schultheiss R. Principles of shock wave therapy. Clin Orthop Relat Res 2001;(387):8-17.

34. Cleveland RO, Chitnis PV, McClure SR. Acoustic field of a ballistic shock wave therapy device. Ultrasound Med Biol 2007;33:1327-35.

35. Furia JP, Rompe JD, Cacchio A, Maffulli N. Shock wave therapy as a treatment of nonunions, avascular necrosis, and delayed healing of stress fractures. Foot Ankle Clin 2010;15:651-62.

36. Csaszar NB, Angstman NB, Milz S, et al. Radial Shock Wave Devices Generate Cavitation. PLoS One 2015;10:e0140541.

37. Huang C, Holfeld J, Schaden W, Orgill D, Ogawa R. Mechanotherapy: revisiting physical therapy and recruiting mechanobiology for a new era in medicine. Trends Mol Med 2013;19:555-64.

38. Holfeld J, Tepekoylu C, Reissig C, et al. Toll-like receptor 3 signalling mediates angiogenic response upon shock wave treatment of ischaemic muscle. Cardiovasc Res 2016;109:331-43.

39. Weihs AM, Fuchs $C$, Teuschl AH, et al. Shock wave treatment enhances cell proliferation and improves wound healing by ATP release-coupled extracellular signal-regulated kinase (ERK) activation. J Biol Chem 2014;289:27090-104.

40. Ueberle F, Delius M, Guo L. Using shock waves for transfer of molecules in cells. Biomed Tech (Berl) 2002;47 Suppl 1 Pt 1:382-5.

41. Cheng $\mathrm{JH}$, Wang $\mathrm{CJ}$. Biological mechanism of shockwave in bone. Int J Surg $2015 ; 24: 143-6$. 
42. Ciampa AR, de Prati AC, Amelio $E$, et al. Nitric oxide mediates antiinflammatory action of extracorporeal shock waves. FEBS Lett 2005;579:683945.

43. Wang CJ, Wang FS, Yang KD, et al. Shock wave therapy induces neovascularization at the tendon-bone junction. A study in rabbits. J Orthop Res $2003 ; 21: 984-9$.

44. Mariotto S, de Prati AC, Cavalieri E, Amelio E, Marlinghaus E, Suzuki H. Extracorporeal shock wave therapy in inflammatory diseases: molecular mechanism that triggers anti-inflammatory action. Curr Med Chem $2009 ; 16: 2366-72$.

45. Chen YL, Chen KH, Yin TC, et al. Extracorporeal shock wave therapy effectively prevented diabetic neuropathy. Am J Transl Res 2015;7:2543-60.

46. Wang CJ, Cheng JH, Chou WY, Hsu SL, Chen JH, Huang CY. Changes of articular cartilage and subchondral bone after extracorporeal shockwave therapy in osteoarthritis of the knee. Int J Med Sci 2017;14:213-23.

47. Holfeld J, Tepekoylu C, Blunder S, et al. Low energy shock wave therapy induces angiogenesis in acute hind-limb ischemia via VEGF receptor 2 phosphorylation. PLoS One 2014;9:e103982

48. Waugh CM, Morrissey D, Jones E, Riley GP, Langberg $H$, Screen HR. In vivo biological response to extracorporeal shockwave therapy in human tendinopathy. Eur Cell Mater 2015;29:268,80; discussion 280.

49. Davis TA, Stojadinovic A, Anam K, et al. Extracorporeal shock wave therapy suppresses the early proinflammatory immune response to a severe cutaneous burn injury. Int Wound J 2009;6:11-21.

50. Abe $\mathrm{Y}$, Ito K, Hao K, et al. Extracorporeal low-energy shock-wave therapy exerts anti-inflammatory effects in a rat model of acute myocardial infarction. Circ ] $2014 ; 78: 2915-25$. 
51. Sun D, Junger WG, Yuan C, et al. Shockwaves induce osteogenic differentiation of human mesenchymal stem cells through ATP release and activation of P2X7 receptors. Stem Cells 2013;31:1170-80.

52. Sukubo NG, Tibalt E, Respizzi S, Locati M, d'Agostino MC. Effect of shock waves on macrophages: A possible role in tissue regeneration and remodeling. Int J Surg $2015 ; 24: 124-30$.

53. de Girolamo L, Stanco D, Galliera E, et al. Soft-focused extracorporeal shock waves increase the expression of tendon-specific markers and the release of antiinflammatory cytokines in an adherent culture model of primary human tendon cells. Ultrasound Med Biol 2014;40:1204-15.

54. Hausdorf J, Schmitz C, Averbeck B, Maier M. Molecular basis for pain mediating properties of extracorporeal shock waves. Schmerz 2004;18:492-7.

55. Maier M, Averbeck B, Milz S, Refior HJ, Schmitz C. Substance $P$ and prostaglandin E2 release after shock wave application to the rabbit femur. Clin Orthop Relat Res 2003;(406):237-45. doi:237-45.

56. Takahashi N, Wada Y, Ohtori S, Saisu T, Moriya H. Application of shock waves to rat skin decreases calcitonin gene-related peptide immunoreactivity in dorsal root ganglion neurons. Auton Neurosci 2003;107:81-4.

57. Saggini R, Di Stefano A, Saggini A, Bellomo RG. Clinical Application of Shock Wave Therapy in Musculoskeletal Disorders: Part i. J Biol Regul Homeost Agents $2015 ; 29: 533-45$.

58. Lee JY, Ha KY, Kim JW, Seo JY, Kim YH. Does extracorporeal shock wave introduce alteration of microenvironment in cell therapy for chronic spinal cord injury? Spine (Phila Pa 1976) 2014;39:E1553-9.

59. Lee JY, Kim SN, Lee IS, Jung H, Lee KS, Koh SE. Effects of Extracorporeal Shock Wave Therapy on Spasticity in Patients after Brain Injury: A Meta-analysis. J Phys Ther Sci 2014;26:1641-7. 
60. Wess OJ. A neural model for chronic pain and pain relief by extracorporeal shock wave treatment. Urol Res 2008;36:327-34.

61. Lohse-Busch H, Marlinghaus E, Reime U, Mowis U. Focused low-energy extracorporeal shock waves with distally symmetric polyneuropathy (DSPNP): a pilot study. NeuroRehabilitation 2014;35:227-33.

62. Kertzman P, Lenza M, Pedrinelli A, Ejnisman B. Shockwave treatment for musculoskeletal diseases and bone consolidation: qualitative analysis of the literature. Rev Bras Ortop 2015;50:3-8.

63. Hu J, Liao H, Ma Z, et al. Focal Adhesion Kinase Signaling Mediated the Enhancement of Osteogenesis of Human Mesenchymal Stem Cells Induced by Extracorporeal Shockwave. Sci Rep 2016;6:20875.

64. Berta L, Fazzari A, Ficco AM, Enrica PM, Catalano MG, Frairia R. Extracorporeal shock waves enhance normal fibroblast proliferation in vitro and activate mRNA expression for TGF-beta1 and for collagen types I and III. Acta Orthop $2009 ; 80: 612-7$.

65. Leone L, Vetrano M, Ranieri D, et al. Extracorporeal Shock Wave Treatment (ESWT) improves in vitro functional activities of ruptured human tendon-derived tenocytes. PLoS One 2012;7:e49759.

66. Zhang D, Kearney CJ, Cheriyan T, Schmid TM, Spector M. Extracorporeal shockwave-induced expression of lubricin in tendons and septa. Cell Tissue Res $2011 ; 346: 255-62$.

67. Kaux JF, Forthomme B, Goff CL, Crielaard JM, Croisier JL. Current opinions on tendinopathy. J Sports Sci Med 2011;10:238-53.

68. Littlewood C, Bateman M, Brown $K$, et al. A self-managed single exercise programme versus usual physiotherapy treatment for rotator cuff tendinopathy: a randomised controlled trial (the SELF study). Clin Rehabil 2016;30:686-96. 
69. Monteleone G, Tramontana A, Mc Donald K, Sorge R, Tiloca A, Foti C. Ultrasonographic evaluation of the shoulder in elite Italian beach volleyball players. J Sports Med Phys Fitness 2015;55:1193-9.

70. de Witte PB, van Adrichem RA, Selten JW, Nagels J, Reijnierse M, Nelissen RG. Persistent shoulder symptoms in calcific tendinitis: clinical and radiological predictors. Ned Tijdschr Geneeskd 2016;160:D521.

71. Del Castillo-Gonzalez F, Ramos-Alvarez JJ, Rodriguez-Fabian G, GonzalezPerez J, Jimenez-Herranz E, Varela E. Extracorporeal shockwaves versus ultrasound-guided percutaneous lavage for the treatment of rotator cuff calcific tendinopathy: a randomized controlled trial. Eur J Phys Rehabil Med 2016;52:14551.

72. Dejaco B, Habets B, van Loon C, van Grinsven S, van Cingel R. Eccentric versus conventional exercise therapy in patients with rotator cuff tendinopathy: a randomized, single blinded, clinical trial. Knee Surg Sports Traumatol Arthrosc 2017;25:2051-9.

73. Louwerens JK, Veltman ES, van Noort A, van den Bekerom MP. The Effectiveness of High-Energy Extracorporeal Shockwave Therapy Versus Ultrasound-Guided Needling Versus Arthroscopic Surgery in the Management of Chronic Calcific Rotator Cuff Tendinopathy: A Systematic Review. Arthroscopy $2016 ; 32: 165-75$.

74. Engebretsen K, Grotle M, Bautz-Holter E, Ekeberg OM, Juel NG, Brox JI. Supervised exercises compared with radial extracorporeal shock-wave therapy for subacromial shoulder pain: 1-year results of a single-blind randomized controlled trial. Phys Ther 2011;91:37-47.

75. Huisstede BM, Gebremariam L, van der Sande R, Hay EM, Koes BW. Evidence for effectiveness of Extracorporal Shock-Wave Therapy (ESWT) to treat calcific and non-calcific rotator cuff tendinosis--a systematic review. Man Ther $2011 ; 16: 419-33$. 
76. Bannuru RR, Flavin NE, Vaysbrot E, Harvey W, McAlindon T. High-energy extracorporeal shock-wave therapy for treating chronic calcific tendinitis of the shoulder: a systematic review. Ann Intern Med 2014;160:542-9.

77. Verstraelen FU, In den Kleef NJ, Jansen L, Morrenhof JW. High-energy versus low-energy extracorporeal shock wave therapy for calcifying tendinitis of the shoulder: which is superior? A meta-analysis. Clin Orthop Relat Res $2014 ; 472: 2816-25$.

78. Wu YC, Tsai WC, Tu YK, Yu TY. Comparative Effectiveness of Nonoperative Treatments for Chronic Calcific Tendinitis of the Shoulder: A Systematic Review and Network Meta-Analysis of Randomized Controlled Trials. Arch Phys Med Rehabil 2017;98:1678,1692.e6.

79. Ioppolo F, Tattoli M, Di Sante $L$, et al. Clinical improvement and resorption of calcifications in calcific tendinitis of the shoulder after shock wave therapy at 6 months' follow-up: a systematic review and meta-analysis. Arch Phys Med Rehabil 2013;94:1699-706.

80. Louwerens JK, Sierevelt IN, van Noort A, van den Bekerom MP. Evidence for minimally invasive therapies in the management of chronic calcific tendinopathy of the rotator cuff: a systematic review and meta-analysis. J Shoulder Elbow Surg $2014 ; 23: 1240-9$.

81. Testa G, Vescio A, Perez S, et al. Extracorporeal Shockwave Therapy Treatment in Upper Limb Diseases: A Systematic Review. J Clin Med 2020;9:10.3390/jcm9020453.

82. Peters J, Luboldt W, Schwarz W, Jacobi V, Herzog C, Vogl TJ. Extracorporeal shock wave therapy in calcific tendinitis of the shoulder. Skeletal Radiol $2004 ; 33: 712-8$.

83. Cacchio A, Paoloni $M$, Barile $A$, et al. Effectiveness of radial shock-wave therapy for calcific tendinitis of the shoulder: single-blind, randomized clinical study. Phys Ther 2006;86:672-82. 
84. Albert JD, Meadeb J, Guggenbuhl P, et al. High-energy extracorporeal shockwave therapy for calcifying tendinitis of the rotator cuff: a randomised trial. J Bone Joint Surg $\mathrm{Br}$ 2007;89:335-41.

85. Speed C. A systematic review of shockwave therapies in soft tissue conditions: focusing on the evidence. Br J Sports Med 2014;48:1538-42.

86. Kolk A, Yang KG, Tamminga R, van der Hoeven H. Radial extracorporeal shockwave therapy in patients with chronic rotator cuff tendinitis: a prospective randomised double-blind placebo-controlled multicentre trial. Bone Joint J 2013;95-B:1521-6.

87. Schofer MD, Hinrichs F, Peterlein CD, Arendt M, Schmitt J. High- versus lowenergy extracorporeal shock wave therapy of rotator cuff tendinopathy: a prospective, randomised, controlled study. Acta Orthop Belg 2009;75:452-8.

88. Galasso O, Amelio E, Riccelli DA, Gasparini G. Short-term outcomes of extracorporeal shock wave therapy for the treatment of chronic non-calcific tendinopathy of the supraspinatus: a double-blind, randomized, placebocontrolled trial. BMC Musculoskelet Disord 2012;13:86,2474-13-86.

89. Bhabra G, Wang A, Ebert JR, Edwards P, Zheng M, Zheng MH. Lateral Elbow Tendinopathy: Development of a Pathophysiology-Based Treatment Algorithm. Orthop J Sports Med 2016;4:2325967116670635.

90. Thiele S, Thiele R, Gerdesmeyer L. Lateral epicondylitis: This is still a main indication for extracorporeal shockwave therapy. Int J Surg 2015;24:165-70.

91. De Smedt T, de Jong A, Van Leemput W, Lieven D, Van Glabbeek F. Lateral epicondylitis in tennis: update on aetiology, biomechanics and treatment. $\mathrm{Br} \mathrm{J}$ Sports Med 2007;41:816-9.

92. Shiri $R$, Viikari-Juntura $E$, Varonen $H$, Heliovaara $M$. Prevalence and determinants of lateral and medial epicondylitis: a population study. Am J Epidemiol 2006;164:1065-74. 
93. Pieber K, Angelmaier L, Csapo R, Herceg M. Acute injuries and overuse syndromes in sport climbing and bouldering in Austria: a descriptive epidemiological study. Wien Klin Wochenschr 2012;124:357-62.

94. Cullinane FL, Boocock MG, Trevelyan FC. Is eccentric exercise an effective treatment for lateral epicondylitis? A systematic review. Clin Rehabil 2014;28:319.

95. Dimitrios S. Lateral elbow tendinopathy: Evidence of physiotherapy management. World J Orthop 2016;7:463-6.

96. Furia JP. Safety and efficacy of extracorporeal shock wave therapy for chronic lateral epicondylitis. Am J Orthop (Belle Mead NJ) 2005;34:13,9; discussion 19.

97. Trentini R, Mangano $T$, Repetto I, et al. Short- to mid-term follow-up effectiveness of US-guided focal extracorporeal shock wave therapy in the treatment of elbow lateral epicondylitis. Musculoskelet Surg 2015;99 Suppl 1:S917.

98. Notarnicola A, Quagliarella L, Sasanelli N, et al. Effects of extracorporeal shock wave therapy on functional and strength recovery of handgrip in patients affected by epicondylitis. Ultrasound Med Biol 2014;40:2830-40.

99. Radwan YA, ElSobhi G, Badawy WS, Reda A, Khalid S. Resistant tennis elbow: shock-wave therapy versus percutaneous tenotomy. Int Orthop 2008;32:671-7.

100. Gunduz R, Malas FU, Borman P, Kocaoglu S, Ozcakar L. Physical therapy, corticosteroid injection, and extracorporeal shock wave treatment in lateral epicondylitis. Clinical and ultrasonographical comparison. Clin Rheumatol $2012 ; 31: 807-12$.

101. Buchbinder R, Green SE, Youd JM, Assendelft WJ, Barnsley L, Smidt N. Systematic review of the efficacy and safety of shock wave therapy for lateral elbow pain. J Rheumatol 2006;33:1351-63. 
102. Rompe JD, Maffulli N. Repetitive shock wave therapy for lateral elbow tendinopathy (tennis elbow): a systematic and qualitative analysis. $\mathrm{Br}$ Med Bull 2007;83:355-78.

103. Rompe JD, Decking J, Schoellner C, Theis C. Repetitive low-energy shock wave treatment for chronic lateral epicondylitis in tennis players. Am J Sports Med $2004 ; 32: 734-43$.

104. Pettrone FA, McCall BR. Extracorporeal shock wave therapy without local anesthesia for chronic lateral epicondylitis. J Bone Joint Surg Am 2005;87:1297304.

105. Chung B, Wiley JP. Effectiveness of extracorporeal shock wave therapy in the treatment of previously untreated lateral epicondylitis: a randomized controlled trial. Am J Sports Med 2004;32:1660-7.

106. Haake M, Konig IR, Decker T, et al. Extracorporeal shock wave therapy in the treatment of lateral epicondylitis : a randomized multicenter trial. J Bone Joint Surg Am 2002;84-A:1982-91.

107. Speed CA, Nichols D, Richards C, et al. Extracorporeal shock wave therapy for lateral epicondylitis--a double blind randomised controlled trial. J Orthop Res $2002 ; 20: 895-8$.

108. Rompe JD, Hopf C, Kullmer K, Heine J, Burger R, Nafe B. Low-energy extracorporal shock wave therapy for persistent tennis elbow. Int Orthop $1996 ; 20: 23-7$.

109. Spacca G, Necozione S, Cacchio A. Radial shock wave therapy for lateral epicondylitis: a prospective randomised controlled single-blind study. Eura Medicophys 2005;41:17-25.

110. Melegati G, Tornese D, Bandi M, Rubini M. Comparison of two ultrasonographic localization techniques for the treatment of lateral epicondylitis with extracorporeal shock wave therapy: a randomized study. Clin Rehabil $2004 ; 18: 366-70$. 
111. Melikyan EY, Shahin E, Miles J, Bainbridge LC. Extracorporeal shock-wave treatment for tennis elbow. A randomised double-blind study. J Bone Joint Surg $\operatorname{Br} 2003 ; 85: 852-5$.

112. Crowther MA, Bannister GC, Huma H, Rooker GD. A prospective, randomised study to compare extracorporeal shock-wave therapy and injection of steroid for the treatment of tennis elbow. J Bone Joint Surg $\operatorname{Br} 2002 ; 84: 678-9$.

113. Yao G, Chen J, Duan Y, Chen X. Efficacy of Extracorporeal Shock Wave Therapy for Lateral Epicondylitis: A Systematic Review and Meta-Analysis. Biomed Res Int 2020;2020:2064781.

114. Yoon SY, Kim YW, Shin IS, Moon HI, Lee SC. Does the Type of Extracorporeal Shock Therapy Influence Treatment Effectiveness in Lateral Epicondylitis? A Systematic Review and Meta-analysis. Clin Orthop Relat Res 2020; .

115. Yan C, Xiong $Y$, Chen $L$, et al. A comparative study of the efficacy of ultrasonics and extracorporeal shock wave in the treatment of tennis elbow: a meta-analysis of randomized controlled trials. J Orthop Surg Res $2019 ; 14: 248,019-1290-y$.

116. Xiong $\mathrm{Y}$, Xue $\mathrm{H}$, Zhou W, et al. Shock-wave therapy versus corticosteroid injection on lateral epicondylitis: a meta-analysis of randomized controlled trials. Phys Sportsmed 2019;47:284-9.

117. Leal C, Ramon S, Furia J, Fernandez A, Romero L, Hernandez-Sierra L. Current concepts of shockwave therapy in chronic patellar tendinopathy. Int J Surg $2015 ; 24: 160-4$.

118. van der Worp $H$, van Ark M, Roerink S, Pepping GJ, van den Akker-Scheek I, Zwerver J. Risk factors for patellar tendinopathy: a systematic review of the literature. Br J Sports Med 2011;45:446-52.

119. Schwartz A, Watson JN, Hutchinson MR. Patellar Tendinopathy. Sports Health $2015 ; 7: 415-20$. 
120. Wang CJ, Ko JY, Chan YS, Weng LH, Hsu SL. Extracorporeal shockwave for chronic patellar tendinopathy. Am J Sports Med 2007;35:972-8.

121. Taunton J, Taunton K, Khan K. Treatment of patellar tendinopathy with extracorporeal shock wave therapy. British Columbia Medical Journal 2003;45.

122. Vulpiani MC, Vetrano M, Savoia V, Di Pangrazio E, Trischitta D, Ferretti A. Jumper's knee treatment with extracorporeal shock wave therapy: a long-term follow-up observational study. J Sports Med Phys Fitness 2007;47:323-8.

123. Zwerver J, Verhagen E, Hartgens F, van den Akker-Scheek I, Diercks RL. The TOPGAME-study: effectiveness of extracorporeal shockwave therapy in jumping athletes with patellar tendinopathy. Design of a randomised controlled trial. BMC Musculoskelet Disord 2010;11:28,2474-11-28.

124. Zwerver J, Hartgens $F$, Verhagen $E$, van der Worp $H$, van den Akker-Scheek I, Diercks RL. No effect of extracorporeal shockwave therapy on patellar tendinopathy in jumping athletes during the competitive season: a randomized clinical trial. Am J Sports Med 2011;39:1191-9.

125. Furia JP, Rompe JD, Cacchio A, Del Buono A, Maffulli N. A single application of low-energy radial extracorporeal shock wave therapy is effective for the management of chronic patellar tendinopathy. Knee Surg Sports Traumatol Arthrosc 2013;21:346-50.

126. Peers KH, Lysens RJ, Brys P, Bellemans J. Cross-sectional outcome analysis of athletes with chronic patellar tendinopathy treated surgically and by extracorporeal shock wave therapy. Clin J Sport Med 2003;13:79-83.

127. Vetrano M, Castorina A, Vulpiani MC, Baldini R, Pavan A, Ferretti A. Plateletrich plasma versus focused shock waves in the treatment of jumper's knee in athletes. Am J Sports Med 2013;41:795-803.

128. van der Worp $\mathrm{H}$, Zwerver J, Hamstra M, van den Akker-Scheek I, Diercks RL. No difference in effectiveness between focused and radial shockwave therapy for treating patellar tendinopathy: a randomized controlled trial. Knee Surg Sports Traumatol Arthrosc 2014;22:2026-32. 
129. Thijs KM, Zwerver J, Backx FJ, et al. Effectiveness of Shockwave Treatment Combined With Eccentric Training for Patellar Tendinopathy: A Double-Blinded Randomized Study. Clin J Sport Med 2017;27:89-96.

130. van der Worp $H$, van den Akker-Scheek $I$, van Schie $H$, Zwerver J. ESWT for tendinopathy: technology and clinical implications. Knee Surg Sports Traumatol Arthrosc 2013;21:1451-8.

131. Moya D, Ramon S, Schaden W, Wang CJ, Guiloff L, Cheng JH. The Role of Extracorporeal Shockwave Treatment in Musculoskeletal Disorders. J Bone Joint Surg Am 2018;100:251-63.

132. Andriolo L, Altamura SA, Reale D, Candrian C, Zaffagnini S, Filardo G. Nonsurgical Treatments of Patellar Tendinopathy: Multiple Injections of PlateletRich Plasma Are a Suitable Option: A Systematic Review and Meta-analysis. Am J Sports Med 2019;47:1001-18.

133. Chen PC, Wu KT, Chou WY, et al. Comparative Effectiveness of Different Nonsurgical Treatments for Patellar Tendinopathy: A Systematic Review and Network Meta-analysis. Arthroscopy 2019;35:3117,3131.e2.

134. Magnan B, Bondi M, Pierantoni S, Samaila E. The pathogenesis of Achilles tendinopathy: a systematic review. Foot Ankle Surg 2014;20:154-9.

135. Kraemer R, Wuerfel W, Lorenzen J, Busche M, Vogt PM, Knobloch K. Analysis of hereditary and medical risk factors in Achilles tendinopathy and Achilles tendon ruptures: a matched pair analysis. Arch Orthop Trauma Surg 2012;132:847-53.

136. Silbernagel KG, Gustavsson A, Thomee R, Karlsson J. Evaluation of lower leg function in patients with Achilles tendinopathy. Knee Surg Sports Traumatol Arthrosc 2006;14:1207-17.

137. Silbernagel KG, Brorsson A, Lundberg $M$. The majority of patients with Achilles tendinopathy recover fully when treated with exercise alone: a 5-year follow-up. Am J Sports Med 2011;39:607-13. 
138. Buda R, Di Caprio F, Bedetti L, Mosca M, Giannini S. Foot overuse diseases in rock climbing: an epidemiologic study. J Am Podiatr Med Assoc 2013;103:11320.

139. Tenforde AS, Sayres LC, McCurdy ML, Collado H, Sainani KL, Fredericson M. Overuse injuries in high school runners: lifetime prevalence and prevention strategies. PM R 2011;3:125,31; quiz 131.

140. Ames PR, Longo UG, Denaro V, Maffulli N. Achilles tendon problems: not just an orthopaedic issue. Disabil Rehabil 2008;30:1646-50.

141. Murphy MC, Travers MJ, Chivers $P$, et al. Efficacy of heavy eccentric calf training for treating mid-portion Achilles tendinopathy: a systematic review and meta-analysis. Br J Sports Med 2019;53:1070-7.

142. Rompe JD, Furia J, Maffulli N. Eccentric loading compared with shock wave treatment for chronic insertional achilles tendinopathy. A randomized, controlled trial. J Bone Joint Surg Am 2008;90:52-61.

143. Gerdesmeyer $L$, Mittermayr R, Fuerst $M$, et al. Current evidence of extracorporeal shock wave therapy in chronic Achilles tendinopathy. Int J Surg $2015 ; 24: 154-9$.

144. Furia JP. High-energy extracorporeal shock wave therapy as a treatment for chronic noninsertional Achilles tendinopathy. Am J Sports Med 2008;36:502-8.

145. Lakshmanan P, O'Doherty D. Chronic achilles tendinopathy: treatment with extracorporeal shock waves. Foot and ankle surgery : official journal of the European Society of Foot and Ankle Surgeons 2004;10:125-30.

146. Rompe JD, Nafe B, Furia JP, Maffulli N. Eccentric loading, shock-wave treatment, or a wait-and-see policy for tendinopathy of the main body of tendo Achillis: a randomized controlled trial. Am J Sports Med 2007;35:374-83.

147. Rompe JD, Furia J, Maffulli N. Eccentric loading versus eccentric loading plus shock-wave treatment for midportion achilles tendinopathy: a randomized controlled trial. Am J Sports Med 2009;37:463-70. 
148. Furia JP. High-energy extracorporeal shock wave therapy as a treatment for insertional Achilles tendinopathy. Am J Sports Med 2006;34:733-40.

149. Costa ML, Shepstone L, Donell ST, Thomas TL. Shock wave therapy for chronic Achilles tendon pain: a randomized placebo-controlled trial. Clin Orthop Relat Res 2005;440:199-204.

150. Rasmussen S, Christensen M, Mathiesen I, Simonson O. Shockwave therapy for chronic Achilles tendinopathy: a double-blind, randomized clinical trial of efficacy. Acta Orthop 2008;79:249-56.

151. Fridman R, Cain JD, Weil L,Jr, Weil LS. Extracorporeal shockwave therapy for the treatment of Achilles tendinopathies: a prospective study. J Am Podiatr Med Assoc 2008; 98:466-8.

152. Vulpiani MC, Trischitta D, Trovato $P$, Vetrano $M$, Ferretti A. Extracorporeal shockwave therapy (ESWT) in Achilles tendinopathy. A long-term follow-up observational study. J Sports Med Phys Fitness 2009;49:171-6.

153. Saxena A, Ramdath S,Jr, O'Halloran P, Gerdesmeyer L, Gollwitzer H. Extracorporeal pulsed-activated therapy ("EPAT" sound wave) for Achilles tendinopathy: a prospective study. J Foot Ankle Surg 2011;50:315-9.

154. Al-Abbad H, Simon JV. The effectiveness of extracorporeal shock wave therapy on chronic achilles tendinopathy: a systematic review. Foot Ankle Int $2013 ; 34: 33-41$.

155. Wiegerinck JI, Kerkhoffs GM, van Sterkenburg MN, Sierevelt IN, van Dijk CN. Treatment for insertional Achilles tendinopathy: a systematic review. Knee Surg Sports Traumatol Arthrosc 2013;21:1345-55.

156. Magnussen RA, Dunn WR, Thomson AB. Nonoperative treatment of midportion Achilles tendinopathy: a systematic review. Clin J Sport Med 2009;19:54-64. 
157. Rathleff MS, Thorborg K. 'Load me up, Scotty': mechanotherapy for plantar fasciopathy (formerly known as plantar fasciitis). Br J Sports Med 2015;49:6389.

158. Monteagudo M, de Albornoz PM, Gutierrez B, Tabuenca J, Alvarez I. Plantar fasciopathy: A current concepts review. EFORT Open Rev 2018;3:485-93.

159. Hansen L, Krogh TP, Ellingsen T, Bolvig L, Fredberg U. Long-Term Prognosis of Plantar Fasciitis: A 5- to 15-Year Follow-up Study of 174 Patients With Ultrasound Examination. Orthop J Sports Med 2018;6:2325967118757983.

160. Trojian T, Tucker AK. Plantar Fasciitis. Am Fam Physician 2019;99:744-50.

161. Lopes AD, Hespanhol Junior LC, Yeung SS, Costa LO. What are the main running-related musculoskeletal injuries? A Systematic Review. Sports Med 2012;42:891-905.

162. Johannsen FE, Herzog RB, Malmgaard-Clausen NM, Hoegberget-Kalisz M, Magnusson SP, Kjaer M. Corticosteroid injection is the best treatment in plantar fasciitis if combined with controlled training. Knee Surg Sports Traumatol Arthrosc $2019 ; 27: 5-12$.

163. Riel H, Jensen MB, Olesen JL, Vicenzino B, Rathleff MS. Self-dosed and predetermined progressive heavy-slow resistance training have similar effects in people with plantar fasciopathy: a randomised trial. J Physiother 2019;65:14451.

164. Almubarak A, Foster N. Exercise Therapy for Plantar Heel Pain: A Systematic Review. International Journal of Exercise Science $2012 ; 5$.

165. Rompe JD, Furia J, Weil L, Maffulli N. Shock wave therapy for chronic plantar fasciopathy. Br Med Bull 2007;81-82:183-208.

166. Gerdesmeyer L, Frey C, Vester J, et al. Radial extracorporeal shock wave therapy is safe and effective in the treatment of chronic recalcitrant plantar fasciitis: results of a confirmatory randomized placebo-controlled multicenter study. Am J Sports Med 2008;36:2100-9. 
167. Chang KV, Chen SY, Chen WS, Tu YK, Chien KL. Comparative effectiveness of focused shock wave therapy of different intensity levels and radial shock wave therapy for treating plantar fasciitis: a systematic review and network metaanalysis. Arch Phys Med Rehabil 2012;93:1259-68.

168. Aqil A, Siddiqui MR, Solan M, Redfern DJ, Gulati V, Cobb JP. Extracorporeal shock wave therapy is effective in treating chronic plantar fasciitis: a metaanalysis of RCTs. Clin Orthop Relat Res 2013;471:3645-52.

169. Zhiyun L, Tao J, Zengwu S. Meta-analysis of high-energy extracorporeal shock wave therapy in recalcitrant plantar fasciitis. Swiss Med Wkly 2013;143:w13825.

170. Dizon JN, Gonzalez-Suarez C, Zamora MT, Gambito ED. Effectiveness of extracorporeal shock wave therapy in chronic plantar fasciitis: a meta-analysis. Am J Phys Med Rehabil 2013;92:606-20.

171. Yin MC, Ye J, Yao M, et al. Is extracorporeal shock wave therapy clinical efficacy for relief of chronic, recalcitrant plantar fasciitis? A systematic review and meta-analysis of randomized placebo or active-treatment controlled trials. Arch Phys Med Rehabil 2014;95:1585-93.

172. Lou J, Wang S, Liu S, Xing G. Effectiveness of Extracorporeal Shock Wave Therapy Without Local Anesthesia in Patients With Recalcitrant Plantar Fasciitis: A Meta-Analysis of Randomized Controlled Trials. Am J Phys Med Rehabil 2017;96:529-34.

173. Sun J, Gao F, Wang Y, Sun W, Jiang B, Li Z. Extracorporeal shock wave therapy is effective in treating chronic plantar fasciitis: A meta-analysis of RCTs. Medicine (Baltimore) 2017;96:e6621.

174. Babatunde OO, Legha A, Littlewood C, et al. Comparative effectiveness of treatment options for plantar heel pain: a systematic review with network metaanalysis. Br J Sports Med 2019;53:182-94.

175. Li X, Zhang L, Gu S, et al. Comparative effectiveness of extracorporeal shock wave, ultrasound, low-level laser therapy, noninvasive interactive 
neurostimulation, and pulsed radiofrequency treatment for treating plantar fasciitis: A systematic review and network meta-analysis. Medicine (Baltimore) 2018;97:e12819.

176. Li H, Lv H, Lin T. Comparison of efficacy of eight treatments for plantar fasciitis: A network meta-analysis. J Cell Physiol 2018;234:860-70.

177. Li H, Xiong $Y$, Zhou W, et al. Shock-wave therapy improved outcome with plantar fasciitis: a meta-analysis of randomized controlled trials. Arch Orthop Trauma Surg 2019;139:1763-70.

178. Hsiao MY, Hung CY, Chang KV, Chien KL, Tu YK, Wang TG. Comparative effectiveness of autologous blood-derived products, shock-wave therapy and corticosteroids for treatment of plantar fasciitis: a network meta-analysis. Rheumatology (Oxford) 2015;54:1735-43.

179. Li S, Wang K, Sun H, et al. Clinical effects of extracorporeal shock-wave therapy and ultrasound-guided local corticosteroid injections for plantar fasciitis in adults: A meta-analysis of randomized controlled trials. Medicine (Baltimore) 2018;97:e13687-.

180. Sun K, Zhou H, Jiang W. Extracorporeal shock wave therapy versus other therapeutic methods for chronic plantar fasciitis. Foot Ankle Surg 2020;26:33-8.

181. Grimaldi A, Fearon A. Gluteal Tendinopathy: Integrating Pathomechanics and Clinical Features in Its Management. J Orthop Sports Phys Ther 2015;45:910-22.

182. Lequesne $M$, Mathieu $P$, Vuillemin-Bodaghi V, Bard H, Djian P. Gluteal tendinopathy in refractory greater trochanter pain syndrome: diagnostic value of two clinical tests. Arthritis Rheum 2008;59:241-6.

183. Grimaldi A, Mellor R, Hodges P, Bennell K, Wajswelner H, Vicenzino B. Gluteal Tendinopathy: A Review of Mechanisms, Assessment and Management. Sports Med 2015;45:1107-19. 
184. Allison K, Salomoni SE, Bennell KL, et al. Hip abductor muscle activity during walking in individuals with gluteal tendinopathy. Scand J Med Sci Sports $2018 ; 28: 686-95$.

185. Plinsinga ML, Coombes BK, Mellor R, et al. Psychological factors not strength deficits are associated with severity of gluteal tendinopathy: A cross-sectional study. Eur J Pain 2018;.

186. Mellor R, Grimaldi A, Wajswelner $H$, et al. Exercise and load modification versus corticosteroid injection versus 'wait and see' for persistent gluteus medius/minimus tendinopathy (the LEAP trial): a protocol for a randomised clinical trial. BMC Musculoskelet Disord 2016;17:196,016-1043-6.

187. Del Buono A, Papalia R, Khanduja V, Denaro V, Maffulli N. Management of the greater trochanteric pain syndrome: a systematic review. $\mathrm{Br}$ Med Bull $2012 ; 102: 115-31$.

188. Furia JP, Rompe JD, Maffulli N. Low-energy extracorporeal shock wave therapy as a treatment for greater trochanteric pain syndrome. Am J Sports Med $2009 ; 37: 1806-13$.

189. Rompe JD, Segal NA, Cacchio A, Furia JP, Morral A, Maffulli N. Home training, local corticosteroid injection, or radial shock wave therapy for greater trochanter pain syndrome. Am J Sports Med 2009;37:1981-90.

190. Carlisi E, Cecini M, Di Natali G, Manzoni F, Tinelli C, Lisi C. Focused extracorporeal shock wave therapy for greater trochanteric pain syndrome with gluteal tendinopathy: a randomized controlled trial. Clin Rehabil 2019;33:670-80.

191. Lempainen L, Johansson K, Banke IJ, et al. Expert opinion: diagnosis and treatment of proximal hamstring tendinopathy. Muscles Ligaments Tendons J $2015 ; 5: 23-8$.

192. Lempainen L, Sarimo J, Mattila K, Vaittinen S, Orava S. Proximal hamstring tendinopathy: results of surgical management and histopathologic findings. Am J Sports Med 2009;37:727-34. 
193. Goom TS, Malliaras P, Reiman MP, Purdam CR. Proximal Hamstring Tendinopathy: Clinical Aspects of Assessment and Management. J Orthop Sports Phys Ther 2016;46:483-93.

194. Benazzo F, Marullo M, Zanon G, Indino C, Pelillo F. Surgical management of chronic proximal hamstring tendinopathy in athletes: a 2 to 11 years of follow-up. J Orthop Traumatol 2013;14:83-9.

195. Cook JL, Purdam C. Is compressive load a factor in the development of tendinopathy? Br J Sports Med 2012;46:163-8.

196. Cacchio A, Rompe JD, Furia JP, Susi P, Santilli V, De Paulis F. Shockwave therapy for the treatment of chronic proximal hamstring tendinopathy in professional athletes. Am J Sports Med 2011;39:146-53.

197. Reilly JM, Bluman E, Tenforde AS. Narrative Review on the Effect of Shockwave Treatment for Management of Upper and Lower Extremity Musculoskeletal Conditions. PM R 2018;

198. Scheuer R, Friedrich M, Hahne J, et al. Approaches to optimize focused extracorporeal shockwave therapy (ESWT) based on an observational study of 363 feet with recalcitrant plantar fasciitis. Int J Surg 2016;27:1-7.

199. Malliaropoulos N, Thompson D, Meke M, et al. Individualised radial extracorporeal shock wave therapy (rESWT) for symptomatic calcific shoulder tendinopathy: a retrospective clinical study. BMC Musculoskelet Disord 2017; 18:513,017-1873-x.

200. Lohrer H, Nauck T, Korakakis V, Malliaropoulos N. Historical ESWT Paradigms Are Overcome: A Narrative Review. Biomed Res Int 2016;2016:3850461.

201. Gaida JE, Cook J. Treatment options for patellar tendinopathy: critical review. Curr Sports Med Rep 2011;10:255-70.

202. Cinar E, Saxena S, Uygur F. Combination Therapy Versus Exercise and Orthotic Support in the Management of Pain in Plantar Fasciitis: A Randomized Controlled Trial. Foot Ankle Int 2018;:1071100717747590. 